\title{
Application of Improved Analysis of Variance to Ionospheric TEC Short-Term Forecast
}

\author{
Yan Gong ${ }^{1}$, Yamin Dang ${ }^{1,2}$ \\ ${ }^{1}$ Chinese Academy of Surveying and Mapping, Beijing, China; ${ }^{2}$ Shandong University of Science and Technology, Qingdao, China. \\ Email: gongy1985@163.com, dangym@casm.ac.cn
}

Received December $8^{\text {th }}$, 2010; revised December 27 ${ }^{\text {th }}$, 2010; accepted January $17^{\text {th }}, 2011$.

\begin{abstract}
An improved superposition analysis of periodical wave variance is used for short-term forecast of the ionosphere TEC in this study. Using the ionospheric TEC data provided by IGS as the real value, the forecasting precision of this method at different locations in China with 40 days data is evaluated. The result shows that the improved method has a better forecasting precision which could reach 1.1 TECU. But the forecasting precision still relates to geographical position, it is proportional to longitude and inversely proportional to latitude. Compared with the current-used methods, the improved method has many advantages as higher precision, using fewer parameters and easier to calculate. So, it applied to ionosphere short-term prediction in China very well.
\end{abstract}

Keywords: Ionosphere, TEC, Short-Term Forecast, Variance Analysis, Cycle Superposition

\section{Introduction}

In order to improve the performance of radio systems, such as communication system, positioning system, radar, navigation et al, and to ensure the safety of space flight, much more precise forecast of ionosphere is becoming indispensable. Short-term forecast which predicts the ionosphere weather change in time scale of days or hours is the development trend [1]. Currently, short-term ionospheric forecast is mainly carried out in America, Europe and Australia et al. with various methods including autocorrelation analysis [2,3], multiple linear regression method [4,5], artificial neural network method [6], the storm-time ionospheric empirical correction model $[7,8]$, interpolation of regional ionospheric forecast [9], equivalent sunspot number method [10,11], ionospheric assimilation model [12,13] et al. Different methods are used in different countries as the ionosphere is different from region to region. So it is necessary to build our own model for short-term forecast of ionosphere which is suited to China.

In China, ionospheric modeling and forecasting plays a key role in this study field. And many models and methods such as Asia region ionospheric F2 layer prediction method [15], Chinese reference ionosphere [16], middle and low latitude $\mathrm{F}$ layer power Numerical simulation of characteristics [17], characteristics of ionospheric region [18] and other methods are developed. But most of them are only capable of long-term forecast rather than short-term. Although some research has been done in short-term forecasting modeling, a practical approach hasn't been achieved so far.

Variance analysis has been studied in this paper, an improved method based on superposition analysis of periodical wave variance has been applied to short-term forecast of ionosperic TEC (Total Electron Content). The forecasting precision of this method was analyzed quantitatively before and after it's improved with reference ionospheric TEC data provided by IGS (International GNSS Service).

\section{Improved Superposition Analysis of Periodical Wave Variance}

Superposition analysis of periodical wave variance is a pure statistical forecast method. The basic principle is to analyze the period of a certain length of processed historical data, and superpose different periods of data together to fit with the historical data, and then extrapolate to make quantitative prediction of the future [19] Ionospheric TEC data was recorded at a particular time with a fixed time interval so can be analyzed as time series.

\subsection{Superposition Analysis of Periodical Wave Variance}

Superposition analysis of periodical wave variance for time series analysis should make time series data arranged with assumption period, if variance within the 
group is less than that between the groups, the arranged period matches to the actual, then the forecasting could be performed. It takes the following three steps to make a prediction.

1) The first cycle analysis.

With time series $X(t)$, assuming there is a periodic vibration of length of $T$, the elements are arranged in $T$ period. If the remaining elements are less than $T$, they will be arranged at the bottom line. Each column is a group and the sums of deviation squares are calculated respectively within and among groups, which are respectively caused by the random error and the difference of mean values of each group. The variance is divided by the respective freedom to obtain the mean square deviation to structure the corresponding $F$ statistics.

The maximum value of $F$ is selected as test period to compare with $F_{0.1}(r-1, n-r)$ which could be checked in the $F$ test critical table. When (if) $F>F_{0.1}(r-1, n-r)$, there is significant difference in the group. After the first cycle measured, the corresponding mean value of each column is as the first cycle component, which could construct a periodic sequence by arranging the mean values in corresponding order.

2) The further cycle analysis.

Remaining series which are the residuals can be obtained by subtracting the first cycle component from the original value. And it needs to be analyzed again with the approach described above. But if $F>F_{0.1}(r-1, n-r)$, a third cycle analysis needs to be done. Follow-up cycles are detected until $F<F_{0.1}(r-1, n-r)$, which means that there is no significant cycle any more.

3) Return test and forecast.

Periodic components would be superposed to obtain the return value of the original series and prediction value could be obtained by extrapolation.

\subsection{Forecasting Model Building and Improving}

In superposition analysis of periodical wave variance, elements of each column minus the corresponding mean value to get the corresponding periodic component after period is detected, which shows all the epoch's contribution is consistent (all the epoch contributes the same) in extrapolation forecast. However, the changes of the ionosphere TEC are not always smooth, which perform increase and decrease, so the contribution about different epochs in the forecast is not same. In order to improve forecasting precision further, each column of the original data is weighted and then the corresponding periodic component is obtained by the weighted values.

To select a certain length of the ionospheric TEC historical data and obtain periodic components by variance analysis, then superimpose periodic components together. When the fitting precision to historical data meets the required level, the quantitative prediction of the trend could be obtained by extrapolation.

Short-term forecasting model of the ionospheric TEC models is as follows:

1) The first cycle analysis.

Suppose there is ionospheric TEC time series:

$$
X(t)=\left\{x_{1}, x_{2}, \cdots, x_{n}\right\}
$$

where, $n$ is the number of epochs. Taking

$T=2,3,4, \ldots,[n / 2]$, the TEC raw data are arranged in the cycle of $T$ and each row is a $T$ cycle:

$$
\begin{array}{ccccc}
x_{1} & x_{2} & x_{3} & \cdots & x_{T} \\
x_{T+1} & x_{T+2} & x_{T+3} & \cdots & x_{2 T} \\
& & \cdots & & \\
x_{(a-1) T+1} & x_{(a-1) T+2} & x_{(a-1) T+3} & \cdots & x_{a T}
\end{array}
$$

where, $a T \leq n$. If $n-a T<T, x_{a T+1}, \cdots, x_{n}$ are arranged at the bottom line. Each column is a group and the sums of deviation squares are calculated respectively within and among groups:

$$
\begin{gathered}
\text { ss } a=\sum_{i=1}^{r} \frac{X_{i}^{\prime 2}}{a}-\frac{1}{n}\left(\sum_{i=1}^{r} X_{i}^{\prime}\right)^{2} \\
\text { sse }=\sum_{i=1}^{r} \sum_{j=1}^{a}{X_{i j}}^{2}-\sum_{i=1}^{r} \frac{X_{i}^{\prime 2}}{a}
\end{gathered}
$$

where, $a$ is the number of columns, $r$ is the number of rows and $X_{i}^{\prime}$ is the sum of each row. The sums of deviation squares are represented sse and ssa respectively within and among groups.

Since ionospheric TEC data are independent, sse and ssa obey the distribution of $\chi^{2}$ and $\mathrm{F}$ statistics constructed:

$$
F=s s a /(r-1) /(\text { sse } /(n-r))
$$

where, $\mathrm{r}-1$ and n-r are the freedom degrees.

The weighted value:

$$
Q=\sum_{\mathrm{j}=1}^{\mathrm{a}} \lambda_{j} x_{(j-1) T+i} \quad(i=1,2, \ldots, T)
$$

The first cycle can get by making the comparison between the max $F$ value and $F_{0.1}(r-1, n-r)$ which can be checked in the $F$ test critical table. The corresponding weighted value of each column is used as the first cycle component, which forms a periodic series by arranging in order. Remaining series could be obtained through the raw series minus the first cycle component.

2) The follow-up cycle analysis.

Remaining series are used as new time series to continue variance analysis by repeating steps 1,2 until no significant cycle. 
3) Return to the raw data test and make prediction of ionospheric TEC.

Superimposing all components of significant periods mentioned above, the test value in return of raw data could be obtained and also the quantitative prediction of future trend by extrapolation.

\section{Examples and Results}

In this paper, the precision of the method improved or not is analyzed through calculating ionospheric TEC data in 2008 provided by IGS, which is selected randomly. Then the calculation of 40 days is taken as the example for analysis. Since the sampling rate of the IGS TEC data is $2.5^{\circ} \times 5^{\circ}$, data calculated with superposition analysis of periodical wave variance including improved and unimproved is selected $\left(\mathrm{N} 30^{\circ}, \mathrm{E} 105^{\circ}\right),\left(\mathrm{N} 35^{\circ}, \mathrm{E} 105^{\circ}\right)$, $\left(\mathrm{N} 40^{\circ}, \mathrm{E} 105^{\circ}\right),\left(\mathrm{N} 30^{\circ}, \mathrm{E} 100^{\circ}\right),\left(\mathrm{N} 30^{\circ}, \mathrm{E}^{\circ}\right),\left(\mathrm{N} 30^{\circ}\right.$, $\left.\mathrm{E} 90^{\circ}\right)$ and $\left(\mathrm{N} 30^{\circ}, \mathrm{E}^{\circ} 5^{\circ}\right)$ from Jan. 11 to Feb. 19 to obtain the prediction of ionospheric TEC about this region in Feb. 20 and Feb. 21 in 2008 by superimposing the cycle components. The calculation about $\left(\mathrm{N} 30^{\circ}, \mathrm{E} 105^{\circ}\right)$ from Jan. 11 to Feb. 19 in 2008 is taken as the example for analysis. Figure 1 shows the results.

This method has an average fitting precision about 0.8 TECU according to multiple experiments, 0.5 TECU is the best. But about $10 \%$ of all the epochs have relatively large error. These epochs are from Beijing Time (BT) 2:00 to 8:00 everyday, which indicates that the improved method has a better performance from 9:00 (BT) to midnight. Obviously in Figure 2, TEC changes regularly from 5:00 to 8:00, irregularly form 8:00 to 12:00 which happen to be 0:00 (BT) to 8:00 (BT). This cannot be just a coincidence, the bad forecasting precision during this

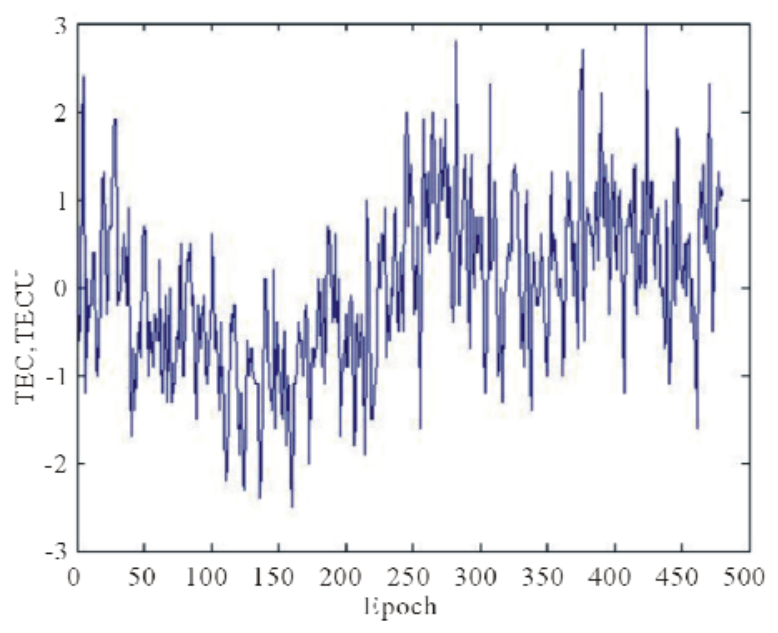

Figure 1. Residual error distribution of observation and pay-back checking value from Jan. 11 to Feb. 19 in 2008.

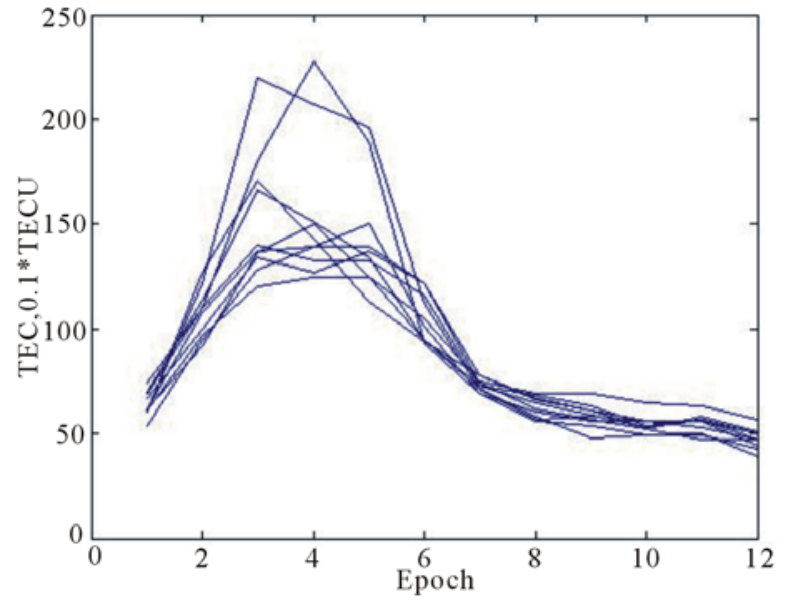

Figure 2. The TEC variation from Jan. 11 to Jan. 20 in 2008.

time period must has something to do with the irregular change of ionosphere. And in this study, it's the main cause of the bad fitting.

A lot of extrapolation experiments have been conducted and here $\left(\mathrm{N} 40^{\circ}, \mathrm{E} 105^{\circ}\right),\left(\mathrm{N} 35^{\circ}, \mathrm{E} 105^{\circ}\right)$ and $\left(\mathrm{N} 30^{\circ}\right.$, $\left.\mathrm{E} 105^{\circ}\right)$ are chosen as examples to show the results. We plot residuals (the forecasted value subtracted the real value from IGS) of these three locations respectively with the original method and the improved method as well (as is shown in Figures 3-5). Clearly, almost all the absolute values of the residuals are no more than 3 TECU except several epochs at N30. And the precision is much better when using the improved method. Wang from Xian University of Electronic Science and Technology gave a prediction precision of 0.75-3.75 TECU in his master thesis by using auto-correlation method. Using the improved method the RMS are 1.537 TECU, 1.258 TECU, 2.292 TECU at the three locations respectively which means this method has good forecasting precision. TEC data at $\left(\mathrm{N} 30^{\circ}, \mathrm{E} 100^{\circ}\right),\left(\mathrm{N} 30^{\circ}, \mathrm{E}^{\circ}\right),\left(\mathrm{N} 30^{\circ}, \mathrm{E} 90^{\circ}\right)$ and $\left(\mathrm{N} 30^{\circ}, \mathrm{E} 85^{\circ}\right)$ has been selected for variance analyzing and extrapolation forecast to explore the relationship between geographical longitude and the forecasting precision of this method, the RMS is shown in Figure 6.

Figure 6 indicates that the forecasting precision is related to longitude and latitude, especially latitude. In the northern hemisphere, the RMS is proportional to longitude and inversely proportional to latitude, which means the precision will decrease in regions with higher longitudes and lower latitudes, and these places are happen to be with intensely changed ionosphere. In addition, Figure 4 shows that the forecasting precision isn't very good at noon in China when the ionosphere changes very severe. Therefore, future studies should focus on how to improve the prediction precision when ionosphere changes intensely. 


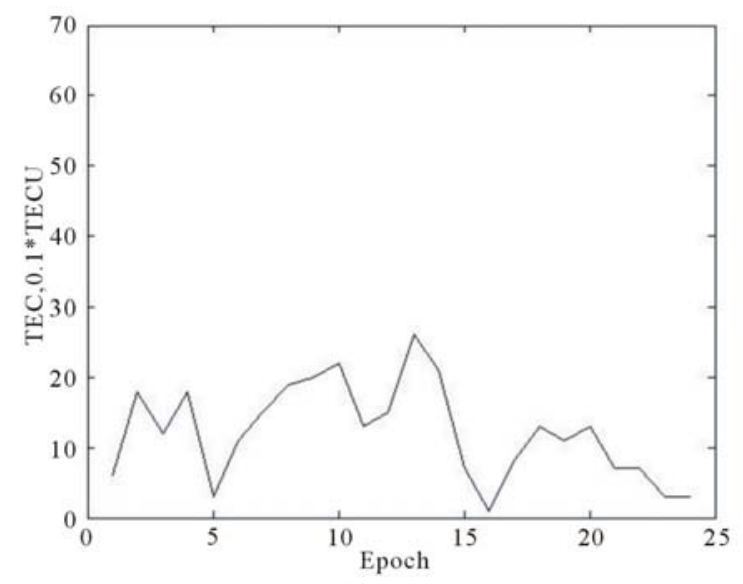

(a)

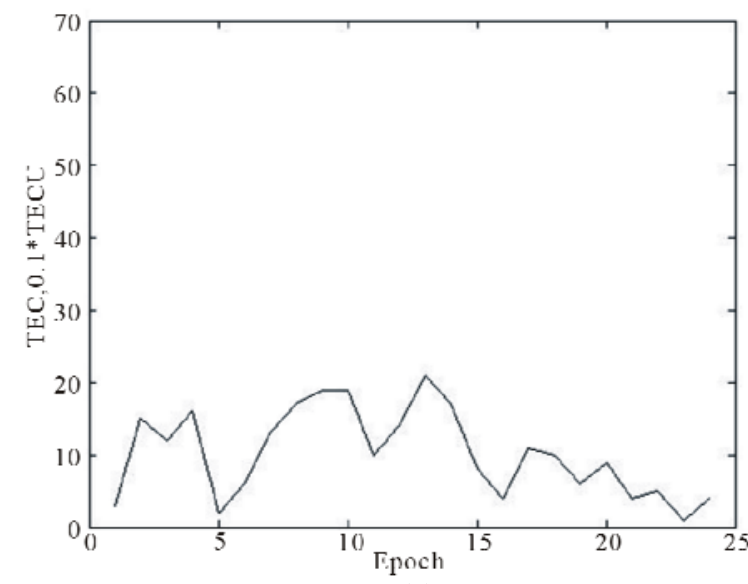

(b)

Figure 3. Residual error plots of original data and forecasting value at (N40 ${ }^{\circ}, \mathrm{E}^{\circ} 5^{\circ}$ ) from Feb. 20 to Feb. 21 in $2008 .($ a) Residual error plot of original method. (b) Residual error plot of improved method.

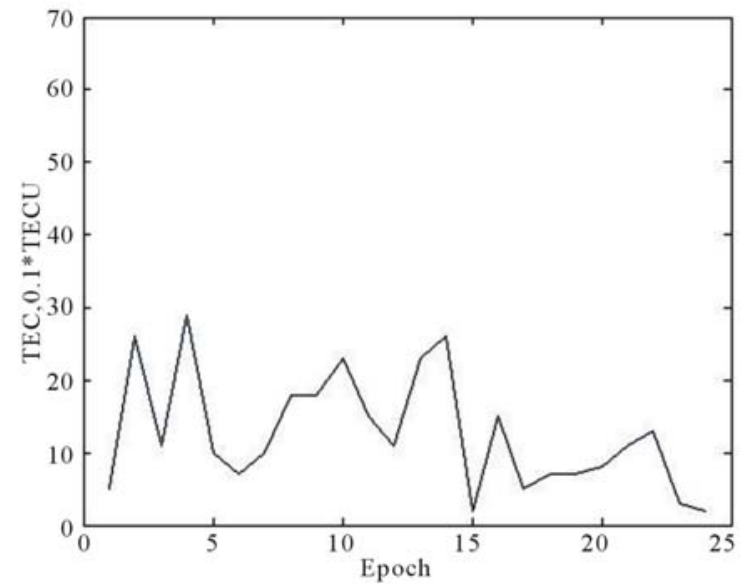

(a)

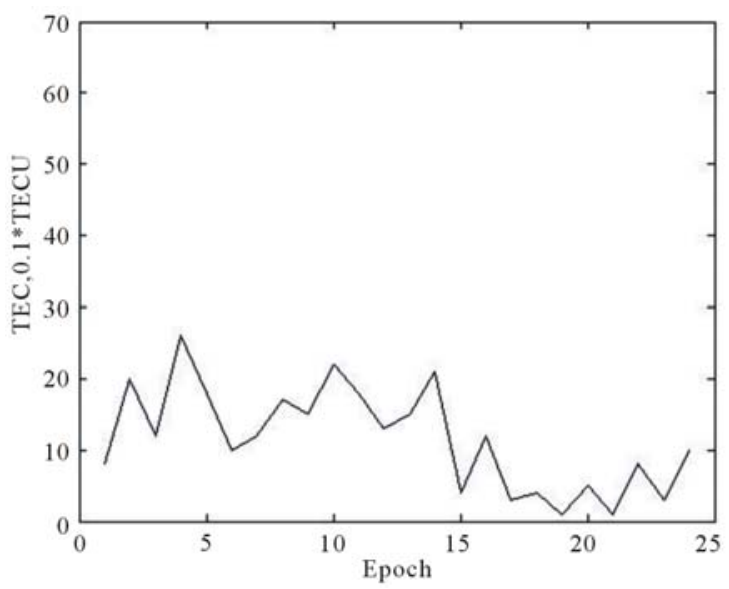

(b)

Figure 4. Residual error plots of original data and forecasting value at (N35 ${ }^{\circ}, \mathrm{E}^{\circ} 5^{\circ}$ ) from Feb. 20 to Feb. 21 in 2008. (a) Residual error plot of original method. (b) Residual error plot of improved method.

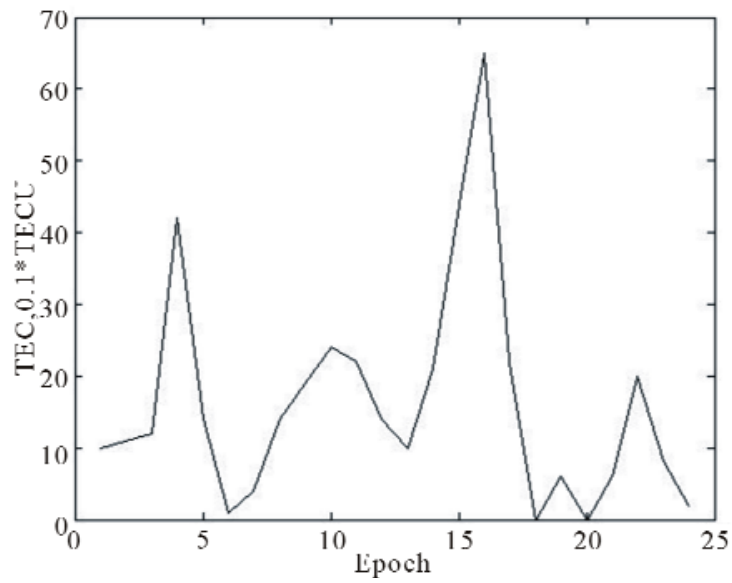

(a)

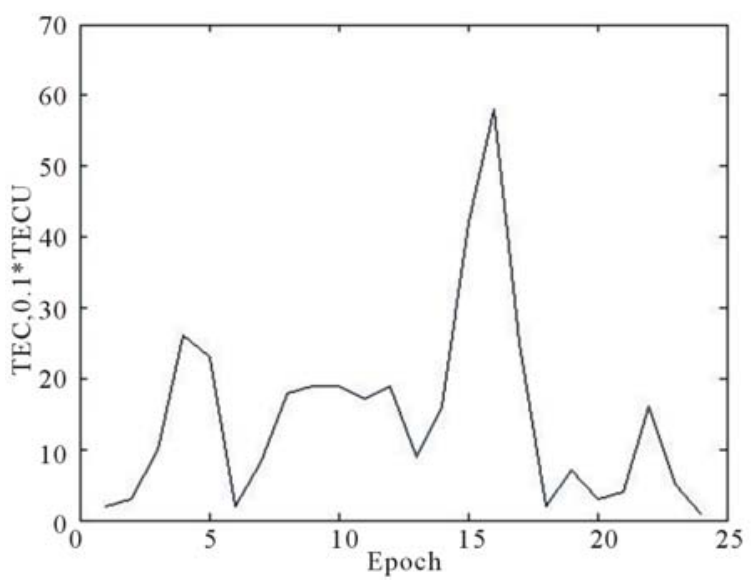

(b)

Figure 5. Residual error plots of original data and forecasting value at (N30 $\left.{ }^{\circ}, \mathrm{E}^{\circ} 5^{\circ}\right)$ from Feb. 20 to Feb. 21 in 2008. (a) Residual error plot of original method. (b) Residual error plot of improved method. 


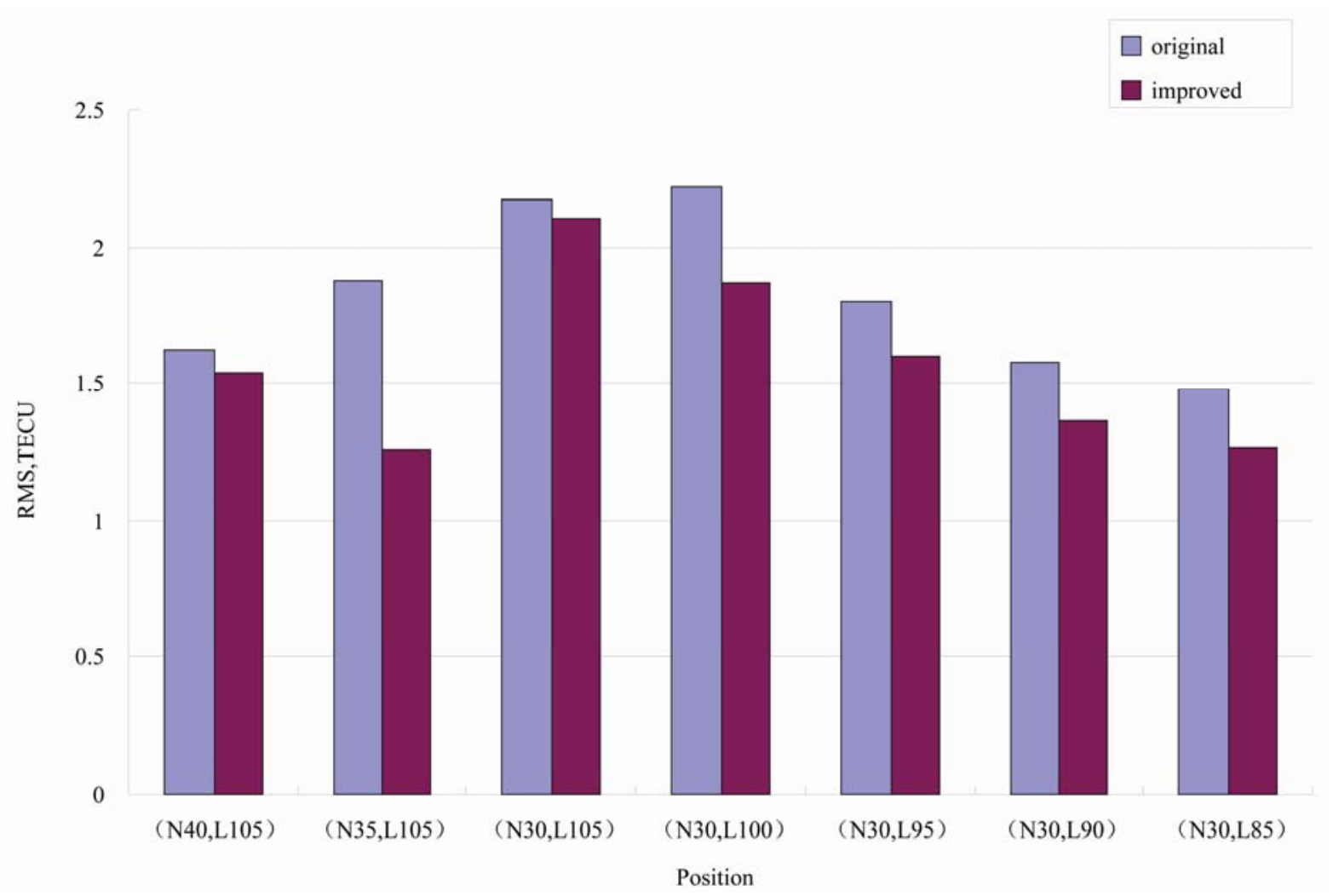

Figure 6. RMS distribution of forecasting value corresponding to each set of data.

\section{Conclusions}

In this paper, superposition analysis of periodical wave variance has been used for ionospheric TEC short-term forecast. Since data at different epoch contributes differently to the extrapolation value, we introduce weighted method to improve this approach. Ionospheric TEC data from different regions with variant time intervals are used in the extrapolation forecast experiments, both the improved method and the original one have been adopted so comparison of the result can be obtained. Statistical analysis shows that the weighted method improves the forecasting precision about 0.2-0.3 TECU. The precision's changing law is different from region to region, and experiments show that our method gives a higher precision in northern and western of China than it does in southern and eastern.

Compared with commonly used methods nowadays, our method provides a higher forecasting precision and also it is relatively simple because no other solarterrestrial physics observations are needed except the raw data. However, it has its own flaws as any other methods, for example, it can't precisely forecast the ionospheric TEC if the ionosphere changes dramatically. So, further improvement needs to be done in the subsequent studies.

\section{REFERENCES}

[1] R. Y. Liu, S. L. Liu, Z. H. Xu et al., “Application of Autocorrelation Method to Ionospheric Short-Term Forecast,” Chinese Science Bulletin, Vol. 50, No. 24, 2005, pp. 2781- 2785.

[2] G. Muhtarov and I. Kutiev, “Autocorrelation Method for Temporal Interpolation and Short-Term Prediction of Ionospheric Data,” Radio Science, Vol. 34, No. 2, 1999, pp. 459-464. doi:10.1029/1998RS900020

[3] R. Hanbaba, “COST 251 Final Report, Improved Quality of Service in Ionospheric Telecommunication Systems Planning and Operation,” Warsaw: Space Research Centre, Vol. 12, 1999, pp. 127-142.

[4] A. V. Mikhailov, B. A. de la Morena, and G. Miro, “A Method for f0F2 Monitoring over Spain Using the El Arenosillo Digisonde Current Observations,” 3rd COST 251 Workshop Proceedings, El Arenosillo, Spain, COST251TD (99)003, 1998, pp. 185-194.

[5] D. Marin, G. Miro and A. V. Mikhailov, "A Method for f0F2 Short-term Prediction,” 4th COST 251 Workshop Proceedings, Madeira, Portugal, COST251TD 008, 1999, pp. 214-222.

[6] Lj. R. Cander, M. Milosavljevic, S. Stankovic, et al., "Ionospheric Forecasting Technique by Artificial Neural Network,” Electron Letter, Vol. 34, No. 6, 1998, pp. 15731574. doi:10.1049/el:19981113 
[7] E. A. Araujo-Pradere, T. J. Fuller-Rowell and M. C. Codrescu, "Storm: An Empirical Storm-Time Ionospheric Correction Model," Model Description. Radio Science, Vol. 37, No. 5, 2002, pp. 3-1-3.12. doi: 10.1029/2001RS- 002467

[8] E. A. Araujo-Pradere, T. J. Fuller-Rowell and M. C. Codrescu, "Srorm: An Emprircal Storm-Time Ionospheric Correction Model, II, Validation,” Radio Science, Vol. 37, No. 5, 2002, pp. 4.1- 4.14. doi: 10.1029/2002RS002620

[9] C. M. Rushm and Jr. W. R. Edwards, “An Authomated Mapping Technique for Representing the Hourly Behavior of The Ionosphere,” Radio Science, Vol. 11, No. 11, 1976, pp. 931-937. doi:10.1029/RS011i011p00931

[10] R. Y. Liu, P. A. Smith and J. W. King, “A New Solar Index Which Leads to Improved $\mathrm{f}_{0} \mathrm{~F} 2$ Predictions Using the CCIR Atlas," Telecommunication Journal, Vol. 50, No. 8, 1983, pp. 408- 414.

[11] J. A. Secan and P. J. Wilkinson, "Statistical Studies of an Effective Sunspot Number,” Radio Science, Vol. 32, No. 4, 1997, pp. 1717- 1724. doi:10.1029/97RS01350

[12] J. J. Sojka, D. C. Thompson, R. W. Schunk, et al., “Assimilation Ionospheric Model: Development and testing with Combined Ionospheric Campain Caribbean measurements,” Radio Science, Vol. 36, No. 2, 2001, pp. 247259. doi:10.1029/1999RS002411

[13] R. W. Schunk, L. Scherliess and J. J. Sojka, "Recent Ap- proaches to Modeling Ionospheric Weather,” Advances in Space Research, 2003, Vol. 31, No. 4, 2003, pp. 819-828.

[14] R. Y. Liu, J. Wu and B. C. Zhang, “Advances in Research of Ionospheric Weather Broadcasting," Chinese Journal of Radio Science, Vol. 19, supplement, 2004, pp. 35-40.

[15] X. R. Sun, “The Asia Oceania Region F_2 Layer Prediction Method," CIC China Communications, Vol. 18, No. 6, 1987, pp. 37-46.

[16] R. Y. Liu, K. H. Quan, K. L. Dai, et al., “A Corrected Method of the International Reference Ionosphere to Be Used in Chinese Region,” ACTA Geophysical Sinica, Vol. 37, No. 4, 1994, pp. 422-432.

[17] J. N. Tu, L. B. Liu and Z. T. Bao, "A Theoretical Low Latitude Ionospheric Model,” Chinese Journal of Space Science, Vol. 17, No. 3, 1997, pp. 212-219.

[18] W. Wan, H. Yuan, B. Ning, et al., "Regional Properties Travelingionospheric Disturbances Observed in Central China," Advances in Space Research, 1999, Vol. 25, No. 1, pp. 219-222. doi:10.1016/S0273-1177(99)00921-7

[19] C. D. Yang, "Broadcast the Annual Rainfall with Superposition Analysis of Periodical Wave Variance," Gui Zhou Atmosphere, Vol. 22, No. 1, 1998, pp. 23-25.

[20] J. P. Wang, "Study of the Short-Term Forecasting of the Ionospheric TEC in China and Its Surrounding Area," XiDian University, Master paper, 2008. 